al País Valencià. Butll. Inst. Cat. Hist. Nat.. 61: 81-82.

SERRA, L., C. FABREGAT, J. JUÁREZ, P. PÉREZ ROVIRA, V. DELTORO, J. PÉREZ BOTELLA, A. OLIVARES, B. PÉREZ ROCHER, M. C. ESCRIBÁ y J. BENITO AYUSO -2000Adiciones y correcciones a la orquidoflora Valenciana, I. Flora Montiberica 15: 10-20.

SOLANAS, J. L. -1998- Flora, vegetació $i$ fitogeografia de la Marina Baixa. Servicio de Publicaciones de la Universidad de Alicante. Alicante.
Aceptado para su publicación en julio de 2000

Dirección de los autores: M.B. CRESPO: Centro Iberoamericano de la Biodiversidad (CIBIO), Universidad de Alicante. Apartado 99. E-03080 Alicante. Correo electrónico: crespo@carn.ua.es. J. PIERA: I.E.S. ${ }^{\circ} 1$ "Bellaguarda". Partida Garganes s/n. E-03590 Altea (Alicante). Correo electrónico: atjopiol@altea.infoville.net. M.R. LOWE: School House, 17. The Village, Brancepeth, Durham DH7 8DG, United Kingdom. Correo electrónico: MichaelRLowe@orchid.freeserve.co.uk.

\title{
89. ADICIONES A LA FLORA DE MURCIA. II
}

\author{
Pedro SÁNCHEZ GÓMEZ, Antonio Félix CARRILLO, Miguel Ángel CARRIÓN, \\ Antonio HERNÁNDEZ y Juan Francisco JIMÉNEZ
}

New records and corrections for the flora of Murcia.

Palabras clave. Flora vascular, corología, Murcia, España.

Key words. Vascular Flora, corology, Murcia, Spain.

Como consecuencia de las nuevas recolecciones realizadas en la provincia de Murcia, han sido localizados una serie de táxones que sirven para mejorar o ampliar los ya incluidos en Flora de Murcia (cf. Sánchez Gómez et al, 1998a),

Asperula cynanchica $\mathrm{L}$.

*MURCIA: Moratalla, Cortijo de la Umbría,
30SWH9424,1040 m, herbazal. M. A. Carrión, J. A. Jiménez, A. F. Carrillo \& P. Sánchez Gómez. 18VI-1998. MGC 48191. Elemento holártico.

Astragalus cavanillesii Podlech

*MURCIA: Moratalla, proximidades del arroyo de la Rogativa, 30SWH6826, $1100 \mathrm{~m}$, herbazal subnitrófilo. C. Selma. 6-VI-1981. MUB20306. Especie hasta el momento tan sólo conocida de La Sagra (Granada). Este taxon ha sido

Trabajo financiado con cargo a los proyectos FLP/9/FS97 de la Fundación SÉNECA y PB97-1061 de la DGES. 
frecuentemente asimilado a Astragalus exscapus $\mathrm{L}$. (Sánchez Gómez et al op. cit.), especie próxima, no presente en la zona. Su área de distribución se circunscribe posiblemente a las sierras béticas del sur de Albacete, norte de Granada y noroeste de Murcia.

\section{Barlia robertiana (Loisel) Greuter}

*MURCIA: Tallante (Cartagena), 30SXG6165, $300 \mathrm{~m}$, pastizales nitrificados sobre suelo pizarroso, J. F. Jiménez. 13-III-1999. MGC 48200. Elemento de distribución circunmediterránea del que sólo se conoce la citada localidad para Murcia.

Cheirolopus mansanetianus Stübing, Peris, Olivares \& Martín

*MURCIA. Alrededores de Cartagena, 30SXG7961, $225 \mathrm{~m}$, matorral sobre litosuelos, J. F. Jiménez. 22-V-1999. MGC 48190. Interesante taxon descrito recientemente. Hasta el momento, además de la localidad típica (Cabo de Gata, Almería), conocemos tan sólo la localidad cartagenera y otra de Cabo Cope (Águilas) (Cueto, com. pers.). Su distribución parece restringida a litosuelos en acantilados próximos al mar, dentro de la provincia biogeográfica Murciano-Almeriense. Este taxon ha sido frecuentemente asimilado a Cheirolopus intybaceus (Lam.) Dostál, del que se distingue en campo por el mayor tamaño de los capítulos. Su relación con otros táxones norteafricanos debe ser estudiada.

\section{Colutea brevialata Lange}

*MURCIA: Abarán, Barranco del Sordo, 30SXH5137, 580 m., matorral, F. J. Martínez Saorín. 19-V-1999. MGC 48193. De acuerdo con la última revisión del género [cf. Talavera in An, Jard. Bot. Madrid (1988) 56 (2) : 410-416] el material murciano tradicionalmente asimilado a Colutea arborescens s. l. corresponde a dos taxones: Colutea hispanica Talavera \& Arista, ya citada para la Sierra de Espuña (Talavera $o p$. cit.), que se encuentra en toda la mitad sur y occidental de la provincia, y Colutea brevialata Lange, presente en las sierras nororientales de Murcia y las más septentrionales de Moratalla, penetrando hacia la provincia de Albacete en las Sierras de Letur y Socovos. No obstante, en la zona de contacto se observan poblaciones de posible origen híbrido.
Crypsis schoenoides (L.) Lam.

*MURCIA: Moratalla, Embalse del Cenajo, 30SXH0547, $400 \mathrm{~m}$, arenas nitrificadas, A. F. Carrillo \& P. Sánchez Gómez. 21-IX-1998. MGC 48197. Elemento euroasiático.

Daphne oleoides Schreb.

*MURCIA: Lorca, Sierra del Gigante, 30SWG8977, $1400 \mathrm{~m}$, matorral sobre sustrato dolomítico, J. M. Egea. 6-VI-1999. MGC48194. Elemento bien representado en las altas montañas calizas béticas, que llega a esta sierra con carácter finícola. Se conoce un solo ejemplar.

Erucastrum nasturtifolium (Poiret) O. E. Schulz

*MURCIA: Moratalla, Cortijo de Tercero 30SWH9529, $700 \mathrm{~m}$, márgenes de caminos. C. Selma. 21-V-1983. MUB 29561. Elemento relativamente frecuente en la zona, cuya recolección había pasado desapercibida.

Juniperus oxycedrus subsp. badia (H. Gay) Debeaux

* MURCIA: Moratalla, Cañada de la Cruz 30SWH6208, $1250 \mathrm{~m}$, entre cultivos de cereales. $P$. Sánchez Gómez, A. F. Carrillo \& E. Muñiz. 27-IX1998. MGC 48192. Elemento iberoafricano presente en páramos y formaciones boscosas con cierta continentalidad. En Murcia se conoce un sólo ejemplar, catalogado como árbol monumental.

Matthiola incana (L.) R. Br. subsp. incana *MURCIA: Cartagena, Castillo San Julián 30SXG7961, $280 \mathrm{~m}$, matorrales nitrófilos cercanos a muros derruidos. P. Sánchez Gómez \& J. F. Jiménez. 24-IV-1999. MGC 48201. Especie ampliamente cultivada que ocasionalmente aparece naturalizada.

Narcissus nevadensis subsp. enemeritoi SánchezGómez, Carrillo, Hernández, Carrión-Vilches \& Güemes

*MURCIA: Moratalla, Sierra de Villafuerte 30SWH72, $1200 \mathrm{~m}$, juncales y pastizales en suelos húmedos. P. Sánchez Gómez \& A. F. Carrillo. 30III-1998. MGC 48195. Taxon recientemente descrito (cf. Sánchez Gómez et al., 1998b) del que se conoce una población de la localidad mencionada de tan solo 130 ejemplares. 
Odontites viscosa (L.) Clairv.

* MURCIA: Moratalla, Sierra del Tejo 30SWH8123, $1200 \mathrm{~m}$, matorrales. P. Sánchez Gómez, A. F. Carrillo \& E. Muñiz. 30-VII-1998. MGC 48184. Taxon frecuente en las sierras béticas de Albacete, por lo que su presencia en Murcia era previsible.

\section{Ononis reclinata $\mathrm{L}$.}

* MURCIA: Cartagena, Castillo San Julián 30SXG7961, $200 \mathrm{~m}$, pedregales, márgenes de caminos. J. F. Jiménez \& A. Hernández. 21-IV1998. MGC 48199. La totalidad de los pliegos de herbario consultados y citas referidas a $O$. reclinata L. dentro del ambito de Murcia, corresponden en el mejor de los casos a $O$. mollis Savi $(O$. reclinata var. mollis (Savi) Halácsy).

\section{Ononis rotundifolia $\mathrm{L}$.}

*MURCIA: Moratalla, Arroyo de Hondares, 30SWH8832, $880 \mathrm{~m}$, juncales y pastizales junto a curso de agua. A. F. Carrillo \& J. F. Jiménez. 25-V1999. MGC 48189. Elemento submediterráneo que se presenta con carácter finícola en las montañas del noroeste murciano.

\section{Orchis purpurea Hudson}

*MURCIA. Moratalla, Carretera Archivel-El Sabinar, 30SWH7924, $1220 \mathrm{~m}$, lastonares. J.A. López Espinosa. 10-V-1999. MGC 47695. Interesante orquídea distribuida por Europa Occidental y la zona mediterránea que llega hasta Murcia con carácter terminal. Las poblaciones más cercanas se encuentran en las montañas béticas de las provincias limítrofes, llegando incluso a la Sierra de Gádor (Almería), (cf. Pallarés, 1999). López Espinosa (com. pers.), recolector de la localidad conocida indica que esta planta es relativamente frecuente en dicha localidad.

\section{Orobanche haenseleri Reut.}

*MURCIA: Moratalla, Rambla de Lucas, 30SWH8133, $1270 \mathrm{~m}$, parásito de Helleborus foetidus L. P. Sánchez Gómez, A. F. Carrillo \& J. F. Jiménez. 4-IV-1999. MGC 48196. Elemento ibérico de distribución poco conocida (Grazalema, Sierra Nevada y Prepirineo) que ha sido recolectado recientemente en el noroeste murciano.

\section{Periploca graeca $\mathrm{L}$.}

*MURCIA: Ulea, proximidades del Balneario de Archena, 30SXH4821, $110 \mathrm{~m}$, naturalizada en bosques riparios. A. F. Carrillo \& P. Sánchez Gómez 10-IX-1998. MGC 48187. Especie del Mediterráneo oriental procedente de cultivo. En la Península Ibérica se conocen varias localidades (Valencia, Lérida,...) en ambientes riparios, al parecer está en fase de rápida expansión.

Peucedanum officinale subsp. stenocarpum (Boiss. \& Reuter) Font Quer

*MURCIA: Alhama de Murcia, Sierra Espuña, 30SXG3091, 780 m, pastizales. P. Sánchez Gómez, M. A. Carrión \& J. F. Jiménez. 30-IX-1998. MGC 48186. Endemismo ibérico propio de montañas con cierta humedad.

\section{Polygala calcarea F.W. Schultz}

*MURCIA: Moratalla, Sierra del Tejo 30SWH8123, $1200 \mathrm{~m}$, juncales y pastizales junto a cursos de agua. P. Sánchez Gómez, A. F. Carrillo \& E. Muñiz. 30-IV-1999. MGC48202. Elemento atlántico-submediterráneo que llega con carácter finícola a las montañas béticas de Murcia.

\section{Polygonum orientale $\mathrm{L}$.}

* MURCIA. Moratalla, El Sabinar, 30SWH7429 $1190 \mathrm{~m}$, asilvestrada en márgenes de viviendas y baldíos. P. Sánchez Gómez, A. F. Carrillo \& E. Muñiz. 2-IX-1998. MGC 48188. Planta que se presenta próxima a lugares habitados, posiblemente procedente de algún cultivo como ornamental.

Quercus canariensis Willd.

*MURCIA. Alhama de Murcia, Sierra Espuña, 30SXG3091, $820 \mathrm{~m}$, fondo de barranco. P. Sánchez Gómez, A. F. Carrillo, J. F. Jiménez \& M. A. Carrión. 22-IX-1998. MGC 48185. Especie introducida que curiosamente no fue mencionada por Esteve (1973). En la actualidad se conocen diversos ejemplares de mediano porte, en barrancos y laderas sombrías en cotas inferiores a los $1000 \mathrm{~m}$. En su conjunto pudieran corresponder a una repoblación coetánea, y la diferencia de tamaño sería consecuencia de la disponibilidad de agua. La mayoría de los árboles no producen frutos, aunque en los sitios más 
favorables comienzan a naturalizarse.

Tragopogon angustifolius Bellardi ex Willd.

* MURCIA: Cartagena, Cabezo de las Colmenas 30SXG8762, $160 \mathrm{~m}$, pastizales nitrificados. J. F. Jiménez \& M. A. Carrión Vilches. 26-IV-1998. MGC48198. Taxon frecuentemente confundido o asimilado a Tragopogon crocifolius $\mathrm{L}$.

\section{BIBLIOGRAFÍA}

ESTEVE, F. -1973-Vegetación y flora de las regiones central y meridional de la provincia de Murcia. Publ. Diputac. Murcia-C.S.I.C.

PALLARÉS NAVARRO, A. -1999- Orquídeas de Almería. Almería, 238 pp.

SÁNCHEZ-GÓMEZ, P., J. GUERRA, E. COY, A. HERNÁNDEZ, S. FERNÁNDEZ y A. F. CARRILLO -1998a- Flora de Murcia. Claves de identificación e iconografía de plantas vasculares. DM Ed. Lérida, 439 pp.
SÁNCHEZ-GÓMEZ, P., J. GUERRA, J. GÜEMES, J. GARCÍA, A. HERNÁNDEZ, A. F. CARRILLO \& M. A. CARRIÓN -1998b- Flora Murciana de Interés Nacional y Europeo. Protección y Legislación. Universidad de Murcia. Consejería de Medio Ambiente, Agricultura y Agua de la Región de Murcia. Fundación SÉNECA. Murcia, 70 pp.

TALAVERA, S. y M. ACOSTA -1998- Notas sobre el género Colutea (Leguminosae) en España. Anales Jard. Bot. Madrid 56 (2):410-416.

Aceptado para su publicación en Octubre de 1999

Dirección de los autores. Departamento de Biología Vegetal (Botánica). Facultad de Biología. Campus de Espinardo. 30100. Murcia. E-mail: psgomez@fcu.um.es

\title{
90. UNA SUBESPECIE NUEVA EN EL GÉNERO SIDERITIS L. (LAMIACEAE)
}

\author{
Manuel Benito CRESPO VILLALBA, José Luís SOLANAS FERRÁNDIZ, \\ Antonio DE LA TORRE GARCÍA y Joaquín PAYÁ MIRA
}

A new subspecies in the genus Sideritis L. (Lamiaceae).

Palabras clave. Sideritis, Lamiaceae, Alicante, España.

Key words. Sideritis, Lamiaceae, Alicante, Spain.

Se propone una nueva subespecie, Sideritis chamaedryfolia Cav. subsp. littoralis M.B. Crespo, Solanas, De la Torre et Payá, después de estudiar el material existente en el
Herbario de la Universidad de Alicante (ABH) recogido en los matorrales sabulícolas de la Sierra Helada ( Alfaz del Pí, Alicante). 